\title{
Towards Robust Automatic Affective Classification of Images Using Facial Expressions for Practical
}

\section{Applications}

\author{
Ligang Zhang ${ }^{\mathrm{a}, \mathrm{b}}$, Dian Tjondronegoro ${ }^{\mathrm{b}}$, Vinod Chandran ${ }^{\mathrm{b}}$ and Jana Eggink ${ }^{\mathrm{c}}$ \\ ${ }^{a}$ Faculty of Computer Science and Engineering, Xi'an University of Technology, Xi'an 710048, China \\ ${ }^{b}$ Science and Engineering Faculty, Queensland University of Technology, Brisbane 4000, Australia \\ ${ }^{\mathrm{c}}$ Research \& Development, British Broadcasting Corporation (BBC), United Kingdom
}

\begin{abstract}
Affect is an important feature of multimedia content and conveys valuable information for multimedia indexing and retrieval. Most existing studies for affective content analysis are limited to low-level features or mid-level representations, and are generally criticized for their incapacity to address the gap between low-level features and high-level human affective perception. The facial expressions of subjects in images carry important semantic information that can substantially influence human affective perception, but have been seldom investigated for affective classification of facial images towards practical applications. This paper presents an automatic image emotion detector (IED) for affective classification of practical (or non-laboratory) data using facial expressions, where a lot of "real-world" challenges are present, including pose, illumination, and size variations. The proposed method is novel, with its framework designed specifically to overcome these challenges using multi-view versions of face and point detectors, and a combination of pointbased texture and geometry. Performance comparisons of several key parameters of relevant algorithms are conducted to explore the optimum parameters for high accuracy and fast computation speed. A comprehensive set of experiments with existing and new datasets, shows that the method is effective despite pose variations, fast, and appropriate for largescale data, and as accurate as the method with state-of-the-art performance on laboratorybased data. The proposed method was also applied to affective classification of images from the $\mathrm{BBC}$ in a task typical for a practical application providing some valuable insights.
\end{abstract}

Keywords Affective Classification, Facial Expression Recognition, Image, Application

\footnotetext{
${ }^{a}$ Corresponding Author. Email addresses: ligzhang@gmail.com, dian@qut.edu.au, v.chandran@qut.edu.au, jana.eggink@bbc.co.uk. Phone: +61 731385074.
} 


\section{Introduction}

The rapid development of information technology has greatly promoted the proliferation of multimedia data, either displayed on-line in the Internet or stored off-line in private repertories. Nearly all major media corporations in the world, such as CNN, Yahoo!, and $\mathrm{BBC}$, have their own data warehouses with a large volume of multimedia data represented in text, image, audio or video formats that are updated every day or even every second. Take YouTube - one of the most popular video sharing website as an example; there are more than 100 hours videos updated every second ${ }^{1}$. The exponential growth of multimedia data put higher demands on developing more effective technologies for multimedia indexing, labeling and classification, to enable people to effectively find and manage media as they wish.

The affect conveyed by image or video content, such as a family photograph, is a valuable source of interesting semantic information for multimedia content analysis. In most cases, viewers might prefer to watch or search data that can arouse their specific emotional experiences or reactions, such as fear or laughter. Many potential applications can be realized if images could be retrieved based on their affective content. Images indexed with emotions could be of immediate use for retrieving memorable moments, for example, the most positive life episodes.

Most existing studies on affective content analysis have either extracted low-level features (e.g. lighting, motion, texture, and color) or constructed middle-level representations (e.g. keywords, fights, and dialogs), which are further mapped into dimensional emotion spaces or categorized emotion classes. These studies are generally criticized for their incapacity to address the affective gap between low-level features and high-level human affective perception [1], which is often subjective and time-varying [2].

High-level features in multimedia content, such as the facial expression of subjects, carry important semantic information that can substantially influence human affective perception. A face with a fear emotion often imposes similar affect on the viewer. However, existing facial expression recognition (FER) systems [3] are often constrained to artificially evoked emotions and strictly controlled environmental conditions. It is still a challenge to develop robust FER systems that are able to work under various variations in practical applications, such as illumination, pose, face size etc. The use of FER for affective

\footnotetext{
${ }^{1}$ http://www.youtube.com/yt/press/statistics.html.
} 
classification of practical data, such as News or website images, has been seldom investigated previously.

This paper presents one of the first pilot studies on affective classification of images using facial expressions in a more challenging environment closer to the wild. There are four major contributions: 1) we present a novel machine-learning based automatic image emotion detector (IED), which builds multi-view versions of face and point detectors combined with point-based texture and geometry features for robustness and accuracy, 2) we perform a series of experimental comparisons on the key parameter of the IED system to achieve an optimized performance with high accuracy and a fast processing speed, both of which are critical for practical applications, 3) we conduct a comprehensive set of experiments with existing and new datasets to prove the robustness to pose variations, fast processing speed and state-ofthe-art performance of the IED system on both realistic and laboratory-based data, and 4) we apply the system into affective classification of images from the $\mathrm{BBC}$, typical for a practical application, providing novel insights into challenges in such applications.

This paper is also an extension and continuation of our previous work [4]. In [4], we compared the FER performance of three texture features (local binary pattern - LBP, scaleinvariant feature transform - SIFT, and Gabor wavelets) and their combinations with facial animation parameters (FAPs) based geometric features, and found that the LBP descriptor is superior to SIFT and Gabor in both the accuracy and computational performance on realistic facial expression data, while SIFT performs the best on laboratory-based data. Thus, the LBP descriptor is adopted in the proposed IED system. Size normalization is also added after face detection to achieve robustness to image size variations. We further exploit several key parameters of the LBP descriptor for obtaining best performance for practical applications.

The rest of the paper is organized as follows. Section 2 reviews related work and Section 3 presents the system framework. The experiments on public datasets and experiments with the BBC practical application are reported in Sections 4 and 5 respectively. Section 6 draws some conclusions.

\section{Related Work}

\subsection{Affective Content Analysis}

Most existing studies on affective content analysis extracted a set of low-level features (e.g. lighting, motion, texture, and color) and mapped it into categorized emotion classes or dimensional emotion spaces (e.g. arousal-valence). These mappings are based on 
either the knowledge learnt from theories of psychology [5], art [1], color, aesthetics, cinematography, etc. [6]; or from employing a train-test process using machine learning algorithms, such as support vector machine (SVM) [7], neural network [8], hidden Markov models, and fuzzy similarity [2]. One of the first affective image classification systems is KDIME [8], which mapped low-level features in images to impression words using a neural network. Machajdik and Hanbury [5] classified images into eight emotional categories based on a set of color, texture, composition and face features extracted from images. Another representative work was conducted by Hanjalic and Qun [9], who modeled arousal and valence intensities linearly and separately using two individual sets of audio-visual features extracted from video. Similar sets of audio-visual features were also used for affect based video recommendation in recent studies [10]. However, these approaches generally suffered from criticisms, such as incapability to bridge the affective gap, which is caused by the lack of correlation between the measurable signal properties (i.e. features) and the expected affective perception of the reviewer [1], as well as the subjective and time-varying problems, which mean that the same image may generate different emotions across different subjects and different time [2]. In short, modeling high-level human perception using low-level features is still a challenge.

To reduce the affective gap, recent studies proposed to construct mid-level feature representations from low-level features that are expected to have a closer link with reviewer affective perception. Acar et al. [11] built mid-level representations from Mel-Frequency Cepstral Coefficients and color values using convolutional neural networks, revealing an improved performance on affective classification of video clips. Liu et al. [7] used the spatial distribution of edges and color harmony, together with a set of low-level features, for affective classification of images. Other statistical bag-of-words methods, such as Latent Dirichlet allocation (LDA) [12] and probabilistic Latent Semantic Analysis (PLSA) [13], have also successfully applied into semantic image classification [14]. Since bag-of-words methods describe only the appearance and ignore the spatial relations of image features, a statistical local spatial relations (SLSR) model was proposed by Han et al. [15] to capture both spatial relations and statistical information. Ionescu et al. [16] predicted mid-level concepts (blood, firearms, fights etc.) for violence detection in Hollywood films. Xu et al. [17] constructed mid-level features to indicate dialog, audio emotional events and textual concepts. Although these representations have shown promising performance to infer highlevel affective content, they still cannot fully reflect user affective understanding of the content. 
High-level features in multimedia content, such as the face and facial expression of subjects, carry important semantic information that can substantially influence human affective perception. In real scenarios, the face in an image often strongly draws the attention of human observers and has shown as a key indication of the affect in the image [5]. The facial expression of the face is also a major way of expressing key ideas and primary moods in multimedia content, and thus it is most likely to evoke similar affective responses from reviewers. Studies (e.g. [18]) have demonstrated that facial expressions contribute $55 \%$ to the effect of the spoken message.

However, current systems of recognizing facial expressions from unconstrained realistic multimedia data are not fully mature, and they have to address a wide range of challenging factors, particularly variations in illumination, pose, face size, and face registration error etc. Although studies [3,19] have attempted to address some of these variations, they are often constrained to artificially evoked emotions and strictly controlled environmental conditions. Facial expressions have not been fully exploited for affective classification of realistic images in a wild environment, and the current literature still lacks systematic investigations on FER systems for practical applications.

\subsection{Facial Expression Representation}

Facial expressions can be generally represented in three ways:

1) Emotion category. This representation method classifies an expression into one of pre-defined categories. The most famous and widely adopted categorical emotions are the six basic emotions - anger (AN), disgust (DI), fear (FE), happiness (HA), sadness (SA), and surprise $(S U)$, which were found to be universal across different cultures and human ethnicities [20]. Aside from basic emotions, non-basic emotions (e.g. interest, disagreement, and pain) are also very important and have particular importance in specific applications, such as driver fatigue monitoring and pain diagnosis.

2) Facial action units (AUs). The AUs are defined in the facial action coding system (FACS) developed by Ekman and Friesen [21] to describe facial expressions. The FACS defines 44 different AUs and each AU may correspond to different facial muscle movements that could generate a certain facial action. The benefit of these AUs is that thousands of expressions and subtle facial signals (e.g. frown and wink) can be expressed by the combination of five dozens of AUs.

3) Dimensional space. The dimensional theory describes emotions using continuous axis values in an $\mathrm{N}$-dimension space, in which each emotion is represented as a point or 
region. This theory is based on the assumption that emotion is best described in terms of latent dimensions rather than discrete categories [22]. One of the most popular 2D spaces is arousal-valence $(\mathrm{A}-\mathrm{V})$ [22], where the arousal axis denotes the level of activation and the valence axis stands for the degree of pleasantness. Dimensional spaces have the advantage of representing a wide range of emotions, and can provide unique insights into the relationship between emotions and emotional intensity. It should be noted that most existing approaches to emotion recognition in dimensional spaces quantize the dimensions into a number of intervals, such as the four quadrants [23], or negative and positive emotions [24], and only few studies [25] have investigated continuous dimensions.

This paper adopts three emotion categories of positive, neutral and negative for the classification experiments, catering for the aim for practical applications where facial expressions are more complicated than pre-defined emotions. Studies [26] have shown that pure expressions of basic emotions are less frequently elicited and blends of emotional displays are often shown by humans in realistic conditions. Consequently, not all emotions that often encounter in social activities can be classified into a pre-defined categorical emotion. Furthermore, ambiguity in interpretation of realistic expressions often exist [4], arising from considerable variations in the intensity and type of emotions, background, facial appearances, and the capacity of expressing facial emotions of subjects etc. By contrast, realistic expressions are easier to be categorized into positive, neutral, and negative. In addition, categories of positive, neutral and negative remain perfectly consistent with the valance axis of the $\mathrm{A}-\mathrm{V}$ dimensional space, providing evidence to their psychological foundations. On the other hand, the AUs are not used here, as they represent expressions via their combinations, which may not have direct linkage or concrete meaning with a specific expression in practical applications.

\subsection{Facial Expression Recognition}

Many FER systems have been proposed previously and a recent comprehensive survey of FER can be found in [3]. In this paper, we limit our focus to reviewing only approaches that fuse texture and geometry, as both of them have been shown to convey important and complementary information for FER [27]. According to the manner of extracting texture features, approaches to fusion of geometry and texture for FER can be approximately classified into holistic, region-based, or point-based as shown in Table 1.

Holistic approaches extract texture features directly from the whole face region. These texture features can be extracted by applying discriminant non-negative matrix factorization 
(DNMF) on difference images [28] or a spatially maximum occurrence model on the face [29]. Geometric features can be represented by grid node coordinate displacements between neutral and expressed frames [28] or the elastic shape-texture matching between neighboring pixels [29]. Active appearance model (AAM) has also been applied to extract and fuse texture and geometry for FER [30][31] (AAM features can also be region-based). These texture features are only suitable for near-frontal faces and often require rigid face registration. The performance deteriorates when face movements, pose and size variations occur.

Table 1

Approaches to fusing texture and geometry.

\begin{tabular}{|c|c|c|c|c|c|}
\hline Approach & Ref. & Texture & Geometry & Database & Nature of data \\
\hline \multirow{4}{*}{ holistic } & {$[28]$} & $\begin{array}{c}\text { DNMF on } \\
\text { differences images }\end{array}$ & $\begin{array}{c}\text { displacements of grid } \\
\text { nodes }\end{array}$ & CK & lab-based \\
\hline & {$[29]$} & $\begin{array}{l}\text { spatially maximum } \\
\text { occurrence }\end{array}$ & $\begin{array}{c}\text { elastic shape-texture } \\
\text { matching }\end{array}$ & AR and Yale & lab-based \\
\hline & {$[30]$} & 2D AAM appearance & 2D and 3D AAM shapes & self-constructed & lab-based \\
\hline & [31] & S-APP and C-APP & S-PTS & UNBC-McMaster & lab-based \\
\hline \multirow{5}{*}{$\begin{array}{l}\text { region } \\
\text { based }\end{array}$} & {$[27]$} & image ratio features & displacements of points & $\begin{array}{l}\text { CK, JAFFE, self- } \\
\text { constructed }\end{array}$ & lab-based \\
\hline & {$[32]$} & surface deformation & angles between points & self-constructed & lab-based \\
\hline & {$[33]$} & wrinkle and furrows & displacement of points & $\begin{array}{l}\text { JAFFE and self- } \\
\text { constructed }\end{array}$ & $\begin{array}{l}\text { lab-based/ } \\
\text { realistic }\end{array}$ \\
\hline & {$[34]$} & appearance of edges & distances between points & Feedtum & lab-based \\
\hline & {$[35]$} & SIFT & spatial distribution of edges & CK & lab-based \\
\hline \multirow{3}{*}{$\begin{array}{l}\text { point } \\
\text { based }\end{array}$} & {$[36]$} & Gabor & coordinates of facial points & JAFFE & lab-based \\
\hline & {$[37]$} & $\begin{array}{l}\text { areas, distances, and } \\
\text { curvatures }\end{array}$ & $\begin{array}{c}\text { Gabor wavelets and } \\
\text { moment }\end{array}$ & self-constructed & lab-based \\
\hline & $\begin{array}{l}\text { This } \\
\text { paper }\end{array}$ & LBP & FAP & $\begin{array}{l}\text { GENKI-4K, QUT FER, } \\
\text { and BBC's ELVIS }\end{array}$ & realistic \\
\hline
\end{tabular}

Region based approaches extract texture features from facial sub-regions defined based on fiducial landmarks that are either manually or automatically pre-located. The texture can be image ratio [27], scale-invariant feature transform (SIFT) [35], surface deformation [32], wrinkles and furrows [33], or appearance of edges [34]. Geometry can be distances, tangent vectors, and angles between landmarks [32], facial animation parameters (FAPs) [27], displacements and angles [33], distances [34], or spatial distributions of edges [35]. These texture features have the advantage of capturing local appearance of the face and retaining some robustness to pose variations. However, questions such as how to define facial regions based on fiducial landmarks and how to extract consistently discriminative features from these regions remain unresolved, as the shapes of the regions are prone to the effects of pose variations and inaccurate locations of landmarks.

Point based approaches extract texture features around fiducial facial points. By taking advantage of the high robustness of facial points to face variations, these approaches achieve more robust features and do not require determination of rules to define facial 
regions. Zhengyou et al. [36] combined point based Gabor features and geometric positions of key points. Peng et al. [37] fused point based Gabor texture and geometric distances and areas for FER on a self-constructed lab-based database. This way of extracting texture has been recommended for FER in practical applications [38].

Nearly all the approaches mentioned earlier are benchmarked on lab-based databases. The use of facial expressions for affective classification of realistic data has not been fully exploited. This paper proposes a point-based emotion detector to address this issue. The facial points are detected by the most widely used Viola-Jones (VJ) detector [39] and the active shape model (ASM) [40], to facilitate fair comparisons with pervious studies. It should be noted that many extensions have been proposed to both the VJ detector and ASM. For instance, the original VJ algorithm has been enhanced from the aspects of feature space (e.g. joint Haar-like feature [41] and adaptive skin-map [42]), classifier learning (e.g. Floatboost [43]), and detector structure (e.g. Width-First-Search tree [44]) etc. A comprehensive review on these extensions can be found in [45]. Similarly, various types of improvements to ASM have also been recorded [46], and recent studies also focus on 3D facial model [47], mixture of facial parts $[48,49]$, and discriminative deep model [50].

\section{System Framework}

\subsection{Challenges for FER in a Practical Environment}

For the purpose of correct recognition of facial expressions under unconstrained practical conditions, several major challenges need to be addressed:

1) pose variation (multiple poses)

2) face registration error (inaccurate face registration)

3) illumination variation (lighting condition)

4) facial size variation (different facial sizes across data) etc.

To solve pose variation, the proposed IED system builds multi-view face detector \& face tracker to address each range of pose angles individually.

To tackle face registration error, the IED extracts texture and geometry features based on fiducial facial points. This way of extracting features has been recommended for FER in practical applications [38].

To handle illumination variation, the IED extracts texture using local binary pattern (LBP) descriptors that have proved robustness to illumination variations, and geometric distances from facial points that are little impacted by illumination variations. 
To ensure robustness against facial size variation, the IED applies size normalization to the facial region and to the geometric distance features.

\subsection{Framework}

Fig. 1 shows a framework for the IED system. For an input image or video frame, the face region and 68 facial points are detected using multi-view versions of the popular ViolaJones (VJ) detector [39] and the ASM [40] respectively. Local binary pattern (LBP) descriptors [51] are extracted around 53 interior facial points and further concatenated into a single vector representing texture features. A subset of the most discriminative features is selected using the minimal redundancy maximal relevance criterion (mRMR) algorithm [52]. Geometric features composed of 43 distances defined using an ASM and FAPs are also extracted. A feature-level fusion of the top LBP texture subset and 43 facial animation parameter (FAP) based distances (denoted as "LBP+FAP") is then employed and a support vector machine $(\mathrm{SVM})$ or regression (SVR) with a RBF kernel is trained for classifying emotions or obtaining the likelihood in each emotion.

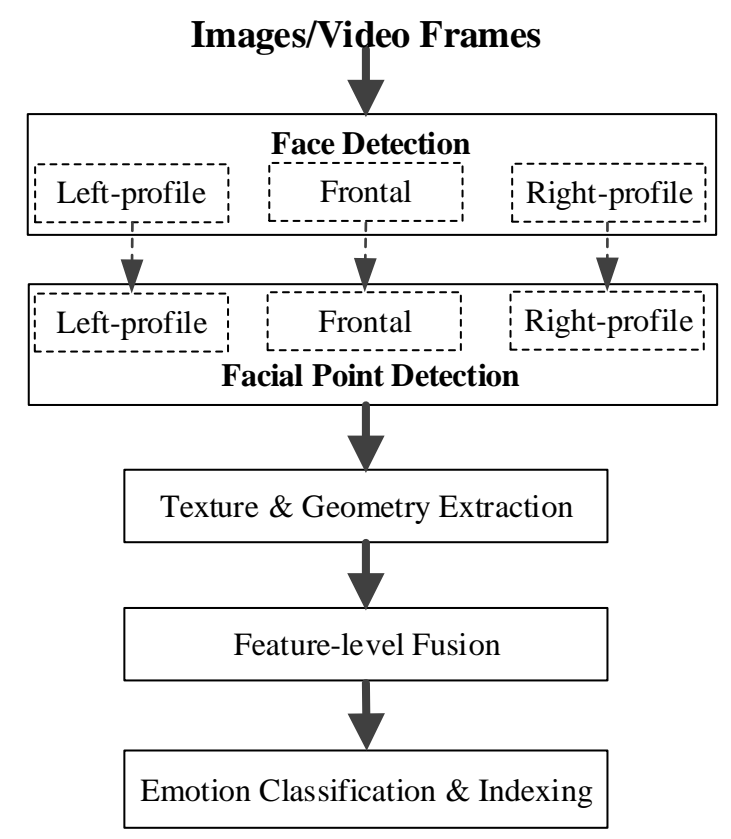

Fig. 1. Framework of the IVED system.

The systematic framework is adopted largely from our previous system [4], which employed a fusion of SIFT and FAP features, and an SVM classifier for FER in the wild. However, there are three improvements to the techniques in the proposed framework: a) the LBP descriptor replaces SIFT for texture feature extraction, as it has shown better overall accuracy and computational performance over SIFT and Gabor features on realistic data [4], 
b) a stage of size normalization is applied to facial regions from the $\mathrm{VJ}$ detector to achieve robustness to the changes of image size, and c) an SVR is included to obtain the probability or intensity of each emotion for indexing practical images.

1) Face and facial point detection. To achieve robustness to pose variations in practical facial images, multi-view versions of the VJ detector and ASM are built to detect facial regions and 68 fiducial facial points as shown in Fig. 2a, respectively. The multi-view VJ detector is built in such a way that the frontal, left and right profile detectors available in OpenCV are applied consecutively when no face has been found by previous detectors. The right profile is implemented by applying the left profile detector on left-right clipped images. To train the multi-view ASM, three image sets were collected from the Internet covering different natural emotions, corresponding to pose ranges of $[-60,20],[-20,20]$, and $[20,60]$ degrees, respectively. Each set has approximate 100 images and a sample set is shown in Fig. 2b. Then 68 points are manually annotated with $\mathrm{x}$ and $\mathrm{y}$ locations, and used to train the ASM for each pose range. To further overcome face size variations in images, the detected facial region is also normalized by scaling the distance between the two eyes to a size of 45 pixels. Therefore, the proposed system is robust to the changes of image resolution provided that the facial region is adequately large in size (i.e. equal or larger than 90 pixels, which is twice the distance between the two eyes).

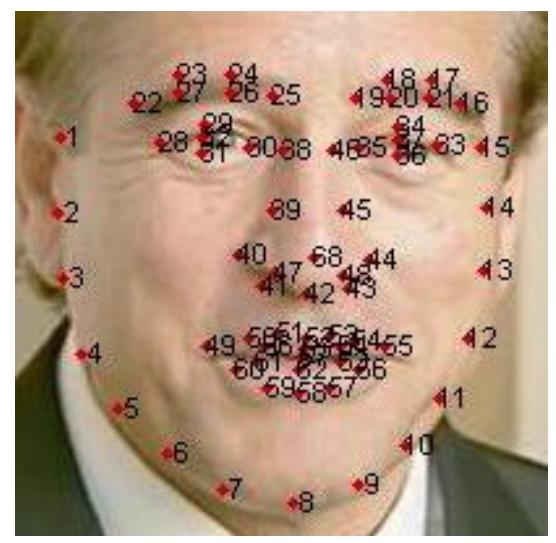

(a)
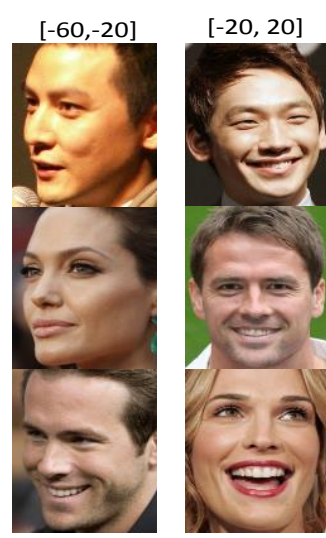

(b)

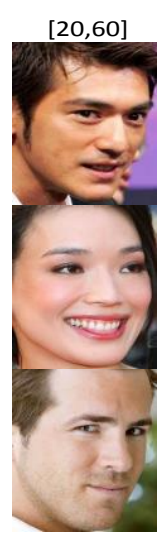

Fig. 2. (a) A set of 68 fiducial points for training the active shape model (ASM); (b) image samples collected from the Internet corresponding to the pose range of $\left[-60^{\circ},-20^{\circ}\right],\left[-20^{\circ}, 20^{\circ}\right]$, and $\left[20^{\circ}, 60^{\circ}\right]$.

2) Texture and geometry feature extraction. Both texture and geometric features are extracted from the local patches around the 53 interior points detected by ASM (index from 16 to 68 in Fig. 2a). The point-based feature extraction helps to maintain a reasonable degree of tolerance to face registration error and pose variations, and has demonstrated robustness to various challenging variations in previous studies [53] and has also been recommended for FER in practical applications [38]. 
Texture: LBP [51] labels each pixel in an image as binary number by applying thresholds to neighborhood pixels with the center value, then accumulates the occurrence of different binary patterns, yielding a histogram as the texture descriptor of the image. Our previous results [54] on spontaneous FER data also show that LBP outperforms SIFT and Gabor wavelet as a feature set in terms of both accuracy and computation. This paper collects uniform patterns $\mathrm{LBP}_{8,2}^{\mathrm{u} 2}$ with 59 histogram bins from a certain size of patches centered at each of 53 points and features of all points are then combined, resulting in a histogram with 3,127 bins as illustrated in Fig. 3. This way of extracting texture features from these points is inspired by previous results [55] that the top LBP features are from facial regions/points around the mouth, nose and eyes.

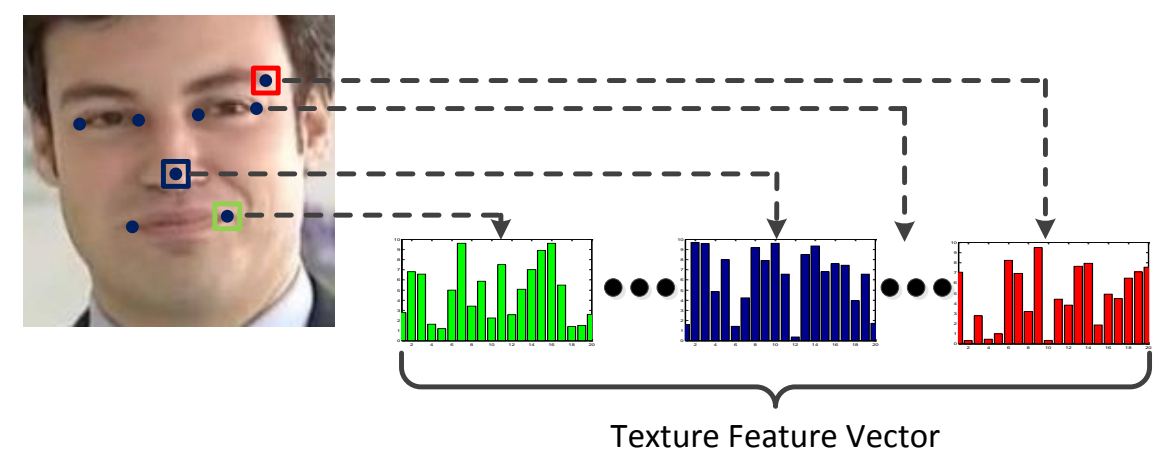

Fig. 3. Construction of the LBP feature vector from 53 facial points.

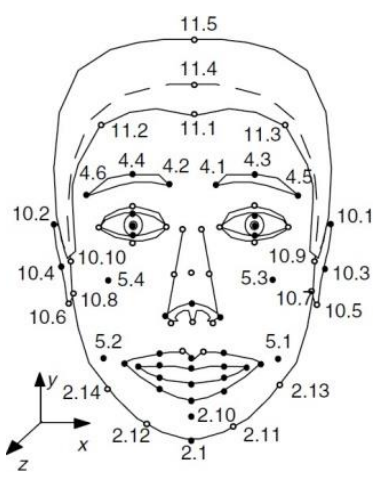

(a)

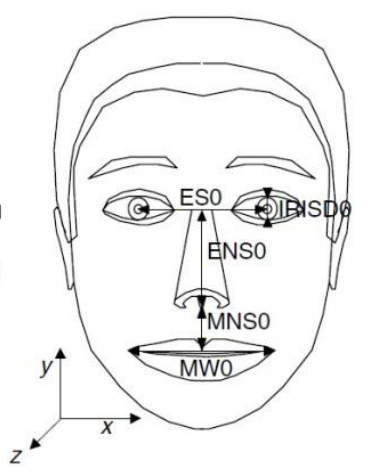

(b)

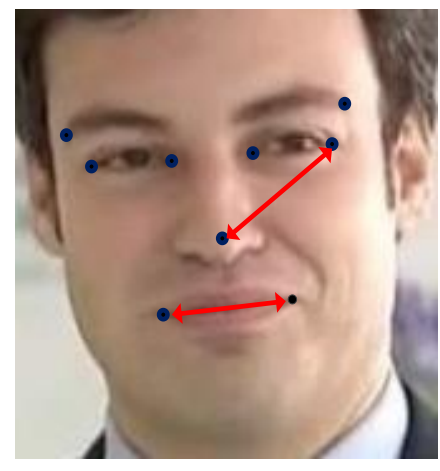

(c)

Fig. 4. (a) A subset of feature points defined in the MPEG-4 FAPs standard, (b) FAPU defined based on fractions of distances between the marked key features, and (c) two examples of the extracted distances.

Geometry: geometry includes 43 distances between 53 interior points, which are directly adopted from [56]. These distances are calculated based on facial animation parameters (FAPs) [57] defined in the ISO MPEG-4 standard (part 2, visual), which have been demonstrated as a sparse, compact, yet information-rich representation of the facial shape [58]. Fig. 4 gives a visual illustration of FAP points and two samples of the extracted distance features. FAPs also can handle arbitrary faces through the use of FAP units and have 
the merits of being robust to translations and rotations of the facial geometry, and do not require compensation for face movements. Therefore, they are suitable for working on realworld data.

3) Discriminative texture feature selection. The mRMR [52] algorithm is used to select a subset of the most discriminative texture features, which has shown better performance over Adaboost and SVM for feature selection on multiple FER databases [54], and has also outperformed PCA, mutual information, and genetic algorithm [59]. From a continuous input feature $D_{k}$, its discrete version $\bar{D}_{k}$ is obtained based on the mean value $\mu_{k}$ and the standard deviation $\sigma_{k}$ of all features:

$$
\bar{D}_{k}=\left\{\begin{array}{rlc}
-2 & \text { if } & D_{k}<\mu_{k}-\sigma * \sigma_{k} \\
0 & \text { if } & \mu_{k}-\sigma * \sigma_{k} \leq D_{k} \leq \mu_{k}+\sigma * \sigma_{k} \\
2 & \text { if } & D_{k}>\mu_{k}+\sigma * \sigma_{k}
\end{array}\right.
$$

where $\sigma$ is set to 0.5 , as recommended in [52].

4) Feature-level fusion. The feature-level fusion strategy is adopted here to combine discriminative texture and geometric feature vectors. The two vectors are normalized individually into $[0,1]$ by dividing by the corresponding maximum value among all elements. The normalized vectors are then concatenated into one fused vector.

5) Emotion classification. The support vector machine (SVM) [60] is a supervised learning algorithm that is widely used for analyzing data and recognizing patterns. It is also arguably the most widely adopted classifier for facial expression analysis in the current literature, and thus the adoption of SVM facilitates fair performance comparisons of our approach with previous approaches using the same SVM classifier. For each image, SVM or support vector regression (SVR) is used to obtain the emotion category or the likelihood in each emotion category. The implementation of a multiple-class SVM or SVR with a RBF kernel in the LIBSVM [61] is adopted (type of SVM/SVR: C-SVC, and parameters: cost C=1 and gamma $=1 /$ num_features). The multiple-emotion-class problem is solved by the oneversus-all strategy for each emotion and the average accuracy over all emotions is used as the final result.

\section{Experiments}

\subsection{Databases}

1) The GENKI-4K [62] is an image dataset that was specifically collected for practical smile detection. It contains 4,000 face images, consisting of 2,162 smile and 1,838 
non-smile, that were labeled by human coders. The majority of the images have a size of around 180x190 pixels with a single face located approximately in the centre and having a pose range of $[-40,40]$ degrees, for both in-plane and out-of-plane rotations. Images from this dataset contain a wide range of variations in image scale, illumination condition, face pose, subject age, gender, occlusion, blurred content, as well as complicated background. Fig. 5 shows a set of image samples with smile and non-smile emotions.

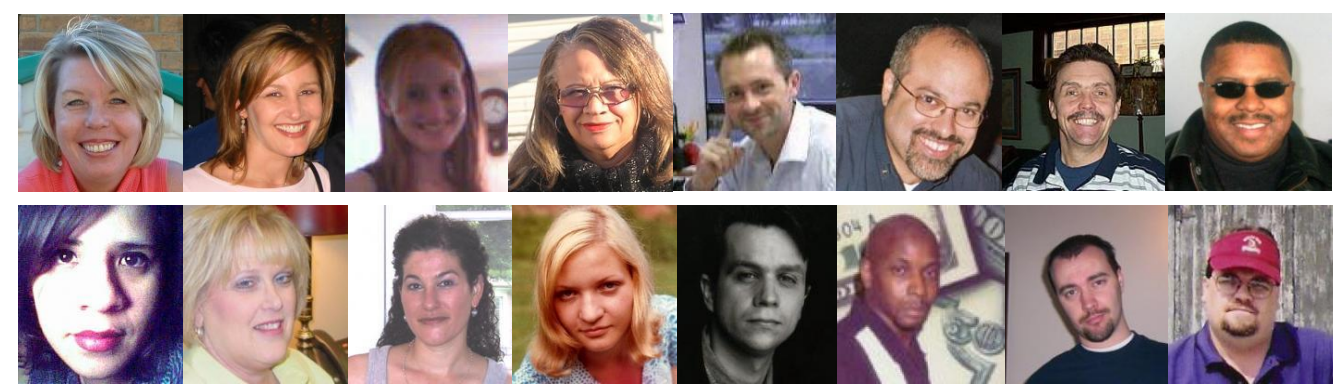

Fig. 5. Smile (top) and non-smile (bottom) samples from the GENKI-4K dataset.

2) The Queensland University of Technology Facial Expression Recognition (QUTFER) dataset [4] comprises of an image subset and a video subset collected from three webbased and broadcast resources: News, TV drama, and YouTube. The image subset is formed by extracting 2-6 typical frames from each video clip to represent facial expressions with different emotion intensities, face poses, and illumination conditions etc., yielding 2,927 images from 219 subjects (102 females and 117 males). All images have the size ranging from $480 \times 360$ to $1024 \times 576$ pixels, and are annotated with (a) six basic emotions plus neutral and (b) positive, neutral, and negative, by five subjects. For the experiment here, 2,489 images annotated with positive, neutral and negative emotions are used (samples shown in Fig. 6).
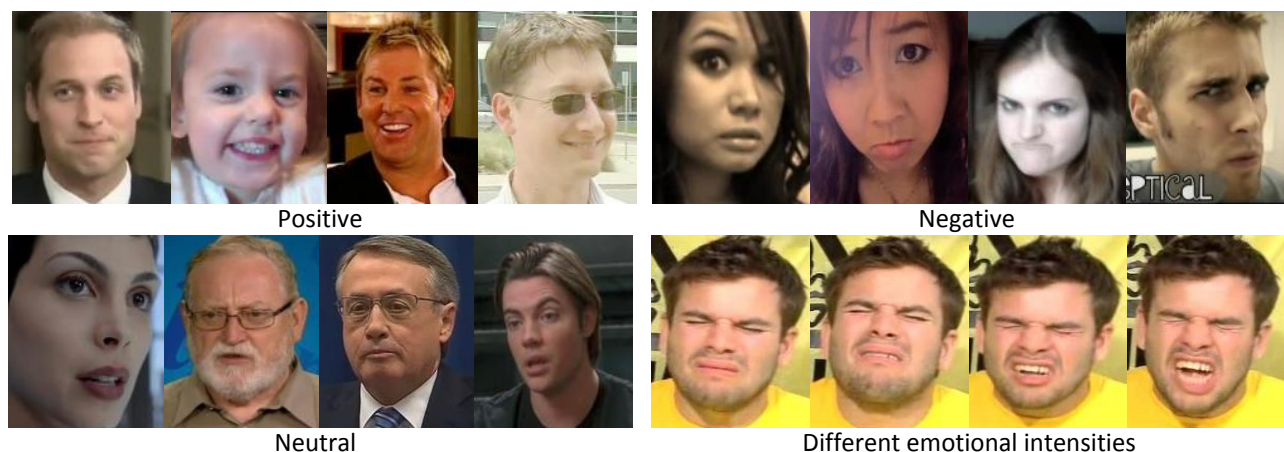

Fig. 6. Image samples from the QUT-FER dataset.

It can be seen that images from both the GENKI-4K and QUT-FER datasets have realistic variations in pose, illumination, and face size etc. They are also expected to have face registration error as the facial region is directly adopted from the results of using Viola- 
Jones and no registration such as rotation of the face to a frontal view and alignment of the face based on accurate location of the eyes is applied to it. There are also other varying factors that cannot be observed directly from the images by eyes, such as compression level and codec formats of the original video resources, but the variations in these factors between images may also exert a certain level of impact on the classification performance. Thus, they are capable of evaluating the robustness of the proposed system under these variations. Table 2 shows the distribution of all images across different types of emotions on the two datasets, and that of successfully detected images after face detection and facial point location stages using the Viola-Jones and ASM.

Table 2

Distribution of the images used in the experiment.

\begin{tabular}{cccccccc}
\hline \multirow{2}{*}{ Image No. } & \multicolumn{2}{c}{ GENKI-4K } & & \multicolumn{3}{c}{ QUT-FER } \\
\cline { 2 - 3 } \cline { 5 - 7 } & Smile & Non-smile & & Positive & Negative & Neutral \\
\hline All & 2,162 & 1,838 & & 1,041 & 1,105 & 343 \\
Detected & 1,964 & 1,649 & & 889 & 849 & 295 \\
\hline
\end{tabular}

\subsection{Comparisons of Key Parameters}

To achieve the best performance of the IED system, this part compares several key parameters of relevant algorithms. The performance is averaged over 10 random subjectindependent cross-validations. To be specific, we first divide all images into different sets in a way that all images from the same subject are included in only one set. Then we randomly select $10 \%$ for the testing set and the other $90 \%$ for the training set, and repeat the process 10 times to generate average performance. The system was developed using Matlab 7.6.0 and Visual Studio 2010 under a laptop configuration of core duo $1.66 \mathrm{GHz} \mathrm{CPU}$ and $2 \mathrm{~GB}$ memory.

1) Dimension of LBP features selected by $m R M R$. Table 3 shows impact of the dimension of LBP features selected by the mRMR algorithm on the performance of the IED system. The computational time is the average cost for a testing image. As can be seen, for both the datasets, a larger dimension of LBP features leads to constantly higher accuracy and longer computation, but the increases on accuracy are not as fast as those on computation. The proposed system has a real-time processing speed on the GENKI-4K dataset and can process more than 10 images per second on the QUT-FER dataset. The big difference in the computation between the two databases is mainly due to the difference in the image size. LBP+FAP has slightly higher accuracy using 200 LBP compared to using 300 LBP features 
on the GENKI-4K dataset. This necessitates choosing a suitable dimension of LBP features that is able to keep the best accuracy and computation. In this paper, the top 200 LBP features selected by the mRMR algorithm are used in the proposed system.

Table 3

Impact of the dimension of LBP features selected by mRMR on classification accuracy $(\%, \pm$ one standard deviation) and computational time (millisecond).

\begin{tabular}{cccccc}
\hline \multirow{2}{*}{ Dim. of LBP } & \multicolumn{2}{c}{ GENKI-4K } & & \multicolumn{2}{c}{ QUT-FER } \\
\cline { 2 - 3 } \cline { 5 - 6 } \cline { 5 - 6 } \cline { 5 - 6 } & Accuracy & Time & & Accuracy & Time \\
\hline 30 & $90.30 \pm 1.49$ & 24.3 & & $83.10 \pm 2.24$ & 86.5 \\
100 & $90.58 \pm 1.31$ & 25.4 & & $86.60 \pm 2.70$ & 87.3 \\
200 & $91.63 \pm 1.37$ & 28.3 & & $87.09 \pm 1.93$ & 89.4 \\
300 & $91.88 \pm 1.33$ & 36.8 & & $\mathbf{9 0 . 7 9} \pm \mathbf{1 . 7 2}$ & 96.7 \\
\hline
\end{tabular}

2) Size of LBP extraction patches. Another important factor for LBP features in the proposed system is the size of point-based facial patches from which LBP features are extracted. Table 4 illustrates the impact of the size of LBP extraction patches on the performance. The top 200 LBP features selected by the algorithm plus 43 FAP features are used. As can be seen, the size of $8 \times 10$ amongst the four sizes produces the highest accuracy for both the two datasets. In addition, it also has the most stable performance on the GENKI$4 \mathrm{~K}$ dataset as demonstrated by one-standard deviation intervals. By contrast, the change of the size also has little impact on the computation. Thus, the size of $8 \times 10$ is used for LBP feature extraction in the proposed system.

\section{Table 4}

Impact of the size of LBP extraction patches on classification accuracy $(\%,+$ one standard deviation) and computational time (millisecond).

\begin{tabular}{cccccc}
\hline \multirow{2}{*}{ Patch size } & \multicolumn{2}{c}{ GENKI-4K } & & \multicolumn{2}{c}{ QUT-FER } \\
\cline { 2 - 3 } \cline { 5 - 6 } \cline { 5 - 6 } \cline { 5 - 6 } & Accuracy & Time & & Accuracy & Time \\
\hline $4 \times 6$ & $90.6 \pm 0.9$ & 31.5 & & $84.3 \pm 2.3$ & 93.6 \\
$6 \times 8$ & $91.3 \pm 1.4$ & 32.4 & & $86.4 \pm 1.9$ & 91.1 \\
$8 \times 10$ & $\mathbf{9 2 . 0} \pm 0.8$ & 33.1 & & $\mathbf{8 8 . 9} \pm 2.4$ & 93.2 \\
$10 \times 12$ & $91.8 \pm 1.7$ & 32.8 & & $88.3 \pm 2.6$ & 96.4 \\
\hline
\end{tabular}

3) Number of LBP extraction points detected by ASM. As the LBP texture features are extracted from patches centered at 53 facial points detected by ASM, features from different points may carry different discrimination capacities for emotion classification. Fig. 7 shows the distribution of the top 200 LBP features over 53 facial points and the visualized spatial distribution on the face. It can be seen that facial points with indices larger than 25 contain nearly all the selected features for smile detection on the GENKI-4K dataset, and a 
significant proportion of the selected features for three emotion classification on the QUTFER dataset. These facial points are mainly distributed in regions of the mouth and nose, while few in regions of the eyes and eyebrows. It is also found that no feature is selected from points with indices of 30,32 , and 53, and this implies that the upper part of the nose region contains little useful features for emotion classification. Based on the distribution frequency of each point, the indices of the top 10 points for GENKI-4K and QUT-FER datasets are $[34,35,39,45,48,41,40,46,49,26]$ and $[34,40,48,39,41,45,49,25,35,46]$, respectively. It is interesting to observe that the two databases share 9 out of the top 10 points (indices: $[34,35,39,45,48,41,40,46,49])$. It seems that the same small set of facial points carries the most discriminative information for FER across different emotion classes and different datasets, and thus, it is advisable to adopt only LBP features from these points.

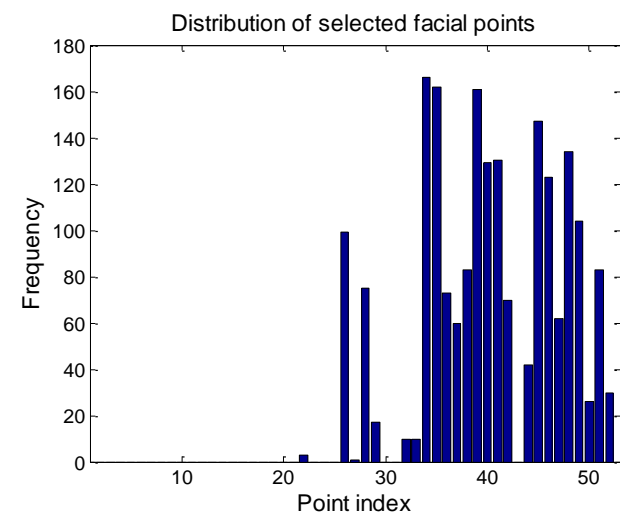

(a) GENKI-4K

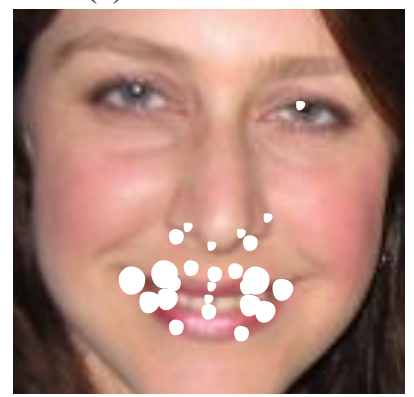

(c) GENKI-4K

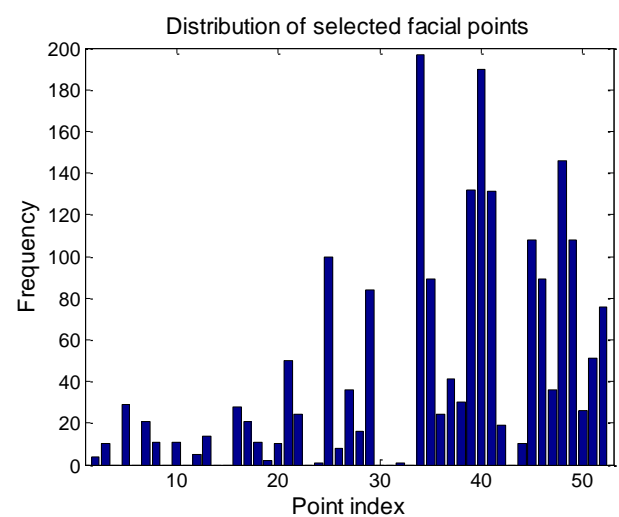

(b) QUT-FER

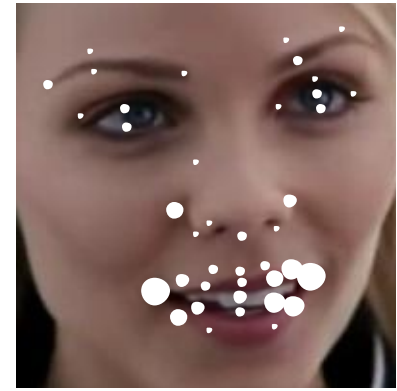

(d) QUT-FER

Fig. 7. (a, b) Distribution of top 200 LBP features over 53 facial points on GENKI-4K and QUT-FER datasets. The indices of all points are mapped to the points in Fig. 2a. (c, d) The corresponding visualized spatial distribution of the facial points in the facial region. A bigger white dot around a point means a larger number of the selected features are distributed at the point.

Table 5 compares the performance obtained using LBP features from the selected top 9 facial points versus from all 53 facial points. It can be seen that using selected points achieves accuracy comparable to using all points on the GENKI-4K dataset, and there is only a $2.7 \%$ accuracy reduction on the QUT-FER dataset, and it is not statistically significant. As for the computational time, substantial reductions (i.e. 15.4 and 34.2 milliseconds) have been 
observed for both the datasets due to using the selected points. It can be concluded that using LBP features from the top 9 points leads to a small accuracy reduction but brings a substantial speed benefit. Thus, the top 9 facial points are used in the proposed system.

Table 5

Performance comparisons of using LBP features from the selected top 9 facial points vs. from all 53 points.

\begin{tabular}{ccccccc}
\hline \multirow{2}{*}{ Facial point } & \multicolumn{2}{c}{ GENKI-4K } & & \multicolumn{2}{c}{ QUT-FER } \\
\cline { 2 - 3 } \cline { 5 - 6 } \cline { 5 - 6 } & Accuracy & Time & & Accuracy & Time \\
\hline All 53 & $92.0 \pm 0.8$ & 33.1 & & $88.9 \pm 2.4$ & 93.2 \\
Top 9 & $91.3 \pm 1.2$ & 17.7 & & $86.1 \pm 1.8$ & 59.0 \\
\hline
\end{tabular}

4) Number of LBP histogram bins. Intuitively, LBP histogram bins are expected to contribute differently to the classification performance. Fig. 8 illustrates the distribution of the top 200 LBP features selected by mRMR over 59 histogram bins. It appears that nearly all bins are making positively contributions to the performance on both the databases. There are also a small proportion of bins that exhibit significantly different frequencies between the two databases, indicating that some bins are of specific significance for classifying different emotion classes (i.e. smile detection vs. three emotions classification). The indices of the top 10 bins for the GENKI-4K and QUT-FER datasets are [11, 34, 21, 15, 16, 14, 7, 43, 56, 51], and $[16,47,56,11,37,44,51,15,53,43]$, respectively. Similar to the results for facial points, the two datasets also share a large proportion of the top LBP histogram bins (i.e. 6 out of 10 points: [11, 15, 16, 43, 56, 51]). Thus, it can be concluded that a small subset of LBP histogram bins and facial points carries the most discriminative information for FER across emotion classes and datasets.

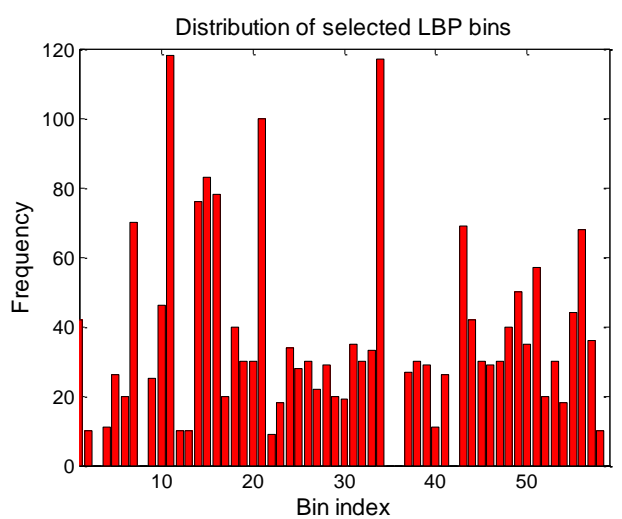

(a) GENKI-4K

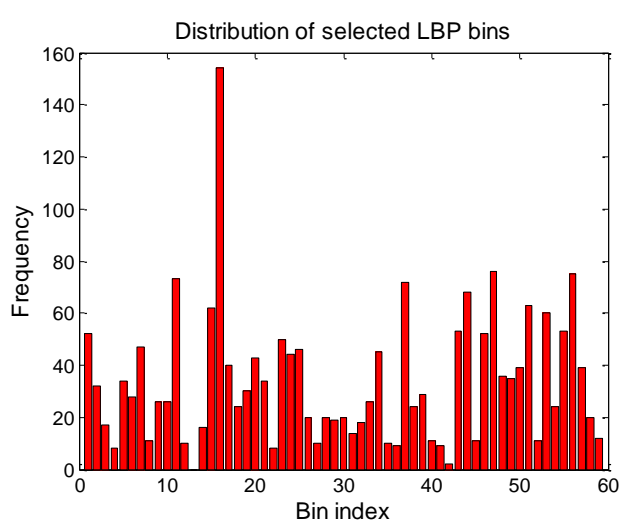

(b) QUT-FER

Fig. 8. Distribution of top 200 LBP features over 59 histogram bins.

Fig. 9 compares the performance obtained using all LBP bins (AllBin), the top 6 bins (SelBin), and the top 6 bins from only the top 9 facial points (SelBin+SelPoint). As can be observed, there are less than 3\% accuracy reductions using the selected top 6 bins compared 
to using all bins on the two datasets, and the performance differences are not statistically significant. However, when the top 6 bins from only the top 9 points are used, the accuracy reductions increase rapidly and are 5\% and $12 \%$ for the GENKI-4K and QUT-FER datasets respectively. The results seem to indicate that the selected LBP bins are able to achieve accuracy comparable to using all bins, but when using the selected bins from only a small set of top facial points, the accuracy declines dramatically. It should note that there is little improvement to the speed, as all bins have to be pre-extracted in these cases. Since the proposed system has already used the top 9 facial points, all LBP histogram bins are adopted here.

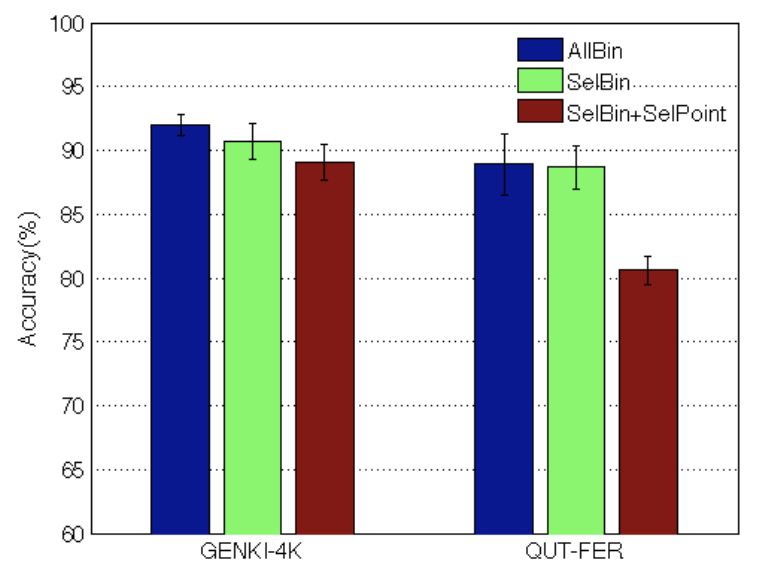

Fig. 9. Performance comparisons of using all LBP bins, the top 6 bins, and the top 6 bins from only the top 9 facial points.

5) Fusion of texture and geometry features. Is it indeed necessary to fuse texture and geometry features for FER in realistic images? Fig.10 compares the classification accuracy between LBP, FAP features and their fusion. It can be seen that fusion of LBP and FAP features leads to slightly higher accuracy (about 1\%) than texture features, but statistically significant higher (more than 20\%) than FAP features. Further, there is nearly no increase in the computation, as the time used for calculating FAP features is almost ignorable compared to that for LBP features (not shown here). The results confirm the benefit to fuse texture and geometry features in the proposed system in terms of accuracy and computation. 

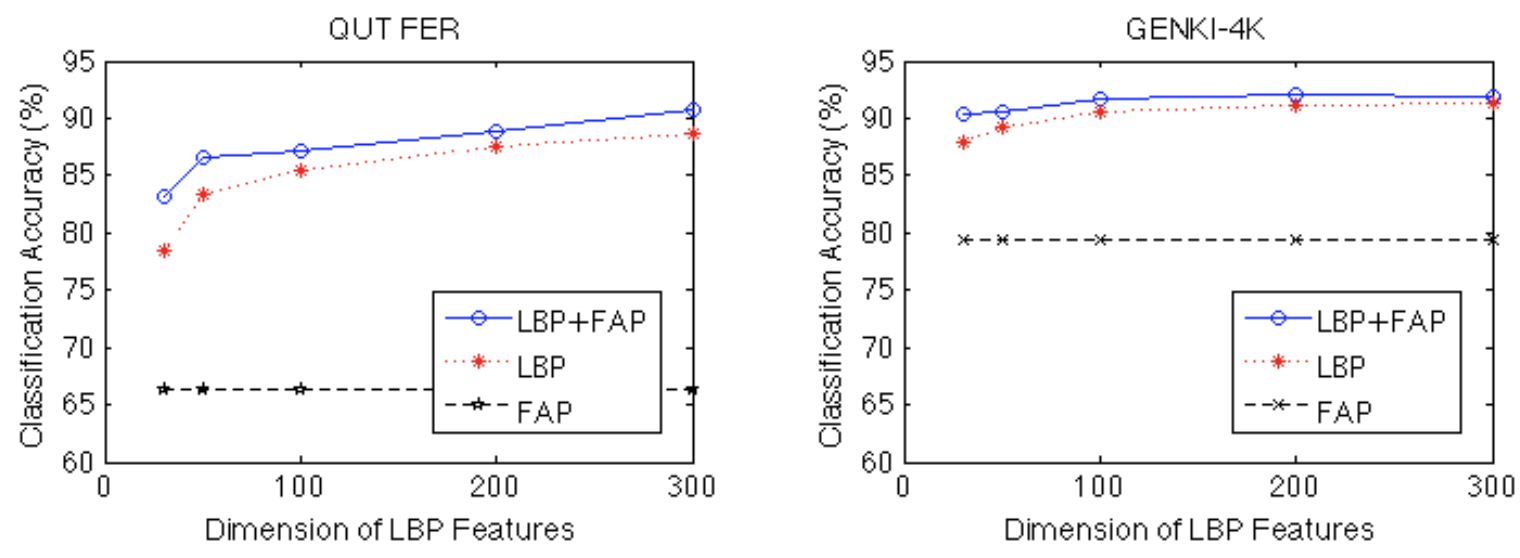

Fig. 10. Performance comparisons of using LBP, FAP features, and their fusion.

\subsection{Performance Evaluation}

The performance of the IED system is tested using images with pose variations and also compared with using SIFT features and previous results.

1) Classification performance under pose variations. Table 6 shows face detection and emotion classification performance of the proposed system evaluated on four groups of GENKI-4K images corresponding to the absolute pose ranges of $\left[0^{\circ}, 5^{\circ}\right],\left[5^{\circ}, 15^{\circ}\right],\left[>15^{\circ}\right]$, and "all", respectively. Note that "all" means using all GENKI-4K images for the tests and it contains all levels of pose variations presented in the dataset. The results are obtained using top 200 LBP features extracted from $8 \times 10$ facial regions plus 43 FAP features. As can be seen, the Viola-Jones and ASM achieve $96.2 \%$ and $94.2 \%$ accuracy for face and point detection under absolute pose ranges of $\left[0^{\circ}, 5^{\circ}\right]$ and $\left[5^{\circ}, 15^{\circ}\right]$ respectively. Although the accuracy suffers from a notable reduction of $15.5 \%$ when the pose range increases to $\left[>15^{\circ}\right]$, it is still higher than $81.7 \%$, showing promising results of the proposed multi-view versions of Viola-Jones and ASM. By contrast, the pose variations have little impact on the classification accuracy, as the accuracies obtained using four image groups are comparable and not significantly different from each other. It should note that using all images leads to the highest accuracy of $92 \%$, and this implies that it is the best to use training images with various levels of pose variations for robust performance. Thus, the IED system has shown promising robustness to pose variations that are expected in practical conditions.

Table 6

Classification performance (\%) under pose variations on the GENKI-4K dataset.

\begin{tabular}{c|cc|cc|cc|c}
\hline Pose (abs.) & \multicolumn{2}{|c|}{$\left[0^{\circ}, 5^{\circ}\right]$} & \multicolumn{2}{c|}{$\left[5^{\circ}, 15^{\circ}\right]$} & \multicolumn{2}{c|}{$>15^{\circ}$} & all \\
\hline & smile & non-smile & smile & non-smile & smile & non-smile & - \\
\hline Image No. & 288 & 239 & 1,220 & 939 & 654 & 660 & 4,000 \\
Detected Image No. & 276 & 231 & 1,157 & 876 & 531 & 542 & 3,613 \\
\hline
\end{tabular}




\begin{tabular}{c|c|c|c|c}
\hline Face Detection Acc. & $\mathbf{9 6 . 2}$ & 94.2 & 81.7 & 90.3 \\
FER Acc. & $91.8 \pm 4.3$ & $91.9 \pm 1.5$ & $89.5 \pm 2.6$ & $\mathbf{9 2 . 0} \pm 0.8$ \\
\hline
\end{tabular}

2) Performance comparisons with SIFT features. Is it a better option to adopt LBP features for FER in realistic images compared to other widely used texture descriptors, such as SIFT? Fig. 11 compares their classification performance on the GENKI-4K and QUT-FER datasets. As can be observed, fusion of LBP and FAP has 5\% higher accuracy than fusion of SIFT and FAP on average on the GENKI-4K dataset. For the QUT-FER dataset, LBP+FAP still outperforms SIFT+FAP before the dimension of texture features reaches 200, after which they tend to perform similarly. With respect to the computation performance, LBP features shows great advantage over SIFT features. For instance, when the top 200 texture features are used, the time required for calculating LBP and SIFT features is 33.1 and 611.5 milliseconds respectively on the GENKI-4K dataset, and 93.2 and 6,626.7 milliseconds respectively on the QUT-FER dataset. Therefore, LBP outperforms SIFT features in both accuracy and computation on the two datasets using the fusion method.
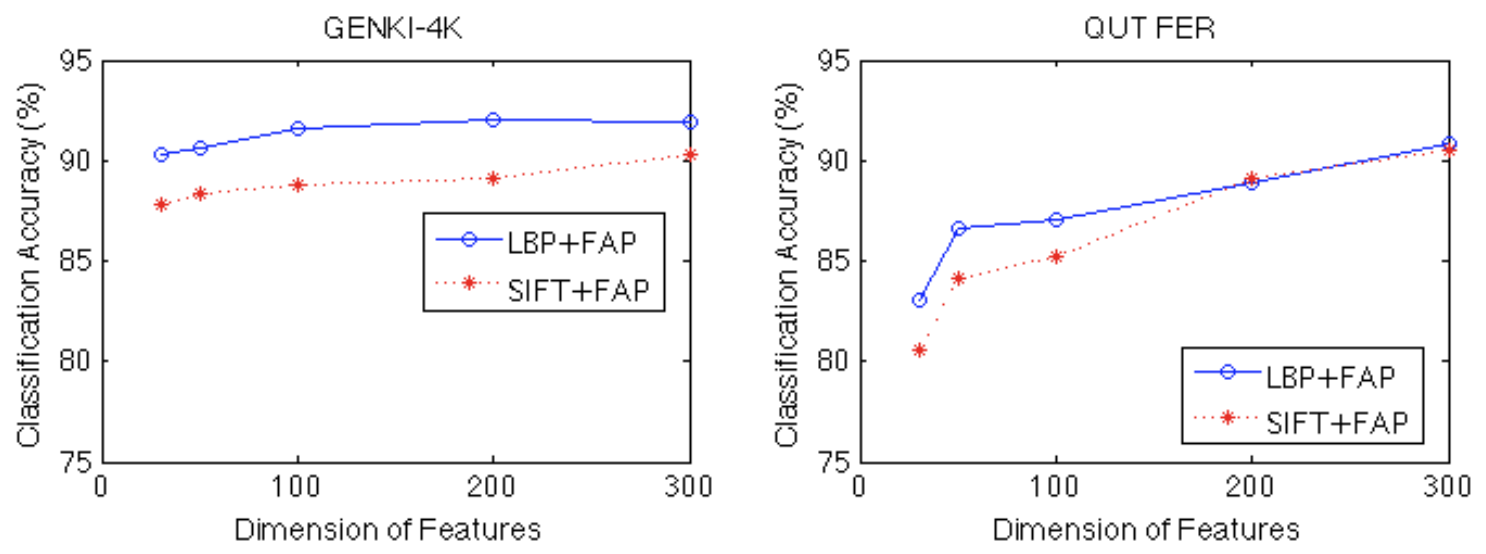

Fig. 11. Performance comparisons of using fused LBP and FAP, and fused SIFT and FAP.

3) Performance comparison with previously reported results. The performance of the proposed system is also compared with previously reported results on both realistic datasets (i.e. GENKI-4K and SFEW) and laboratory-based dataset (i.e. NVIE) as shown in Table 7. For fair comparisons, the same evaluation protocols defined in the compared previous studies are used to obtain the performance of the proposed system. For compared studies that have multiple classification results, only the highest accuracy is used for the comparisons. Note that the results in [63] are based on manually registered faces in four-fold cross validations, and those in [62] are based on registered faces using an automatic eye finder. 
When evaluated on the GENKI-4K dataset, the proposed system using LBP+FAP and SVM has $7.8 \%$ and $2.3 \%$ higher accuracy than the approaches using LBP and SVM in [64] and using pixel intensity differences (PID) and Adaboost classifier in [63], and maintains a similar performance to those approaches using Pixel Mask and ANN in [65] and using HOG and SVM in [66]. Its performance is $1.7 \%$ and $4.3 \%$ lower than those obtained using LBP and GEF features and SVM classifier in [62], which may be due to the fact that it extracts features from only 53 facial points, as opposed to these approaches that use features from the whole face region. Thus, useful features that exist in other facial regions are not utilized in the proposed system. For the SFEW-SPI dataset, there is an improvement of $7.14 \%$ compared with the baseline accuracy of $19.0 \%$ obtained using combined LPQ and PHOG features and a non-linear SVM classifier in [67]. Its performance is competitive to the best result of the HOG and SVM approach [66], with enhanced training by constructing reference manifolds and performing semi-supervised clustering on unlabeled data. The proposed system also outperforms the BeFIT workshop baselines in [67] in the aspects of precision, recall and specificity for classifying seven emotions as shown in Table 8. For laboratory-based data, the proposed system outperforms all compared previous studies in [68] [69] [70] using NVIE images. It should be noted that our approach is the only one, among all benchmarked approaches, that does not require face registration and does not put any requirement on pose, face size and illumination etc. By contrast, the features used in these previous studies are generally based on the assumption of a normalized face with a frontal view and little in-plane or out-of-plane rotations. Thus, the proposed system achieves state-of-the-art performance on both realistic and laboratory-based datasets.

Table 7

Classification performance (\%) comparisons with previously reported results.

\begin{tabular}{|c|c|c|c|c|c|c|}
\hline & & Ref. & Feature \& Classifier & Reg. & No. & Acc. \\
\hline \multirow{12}{*}{ Realistic } & \multirow{10}{*}{ GENKI-4K } & Our & LBP+FAP \& SVM & $x$ & 2 & 92.0 \\
\hline & & & LBP \& SVM & & & 84.2 \\
\hline & & [64] & LPQ \& SVM & $\sqrt{ }$ & 2 & 84.4 \\
\hline & & & HOG \& ELM & & & 88.2 \\
\hline & & [63] & PID \& Adaboost & $\sqrt{ }$ & 2 & 89.7 \\
\hline & & {$[65]$} & Pixel Mask \& ANN & $\sqrt{ }$ & 2 & 92.0 \\
\hline & & {$[66]$} & HOG \& SVM & & 2 & 92.3 \\
\hline & & & LBP \& SVM & & & 93.7 \\
\hline & & {$[62]$} & GEF \& SVM & $\sqrt{ }$ & 2 & 96.3 \\
\hline & & & BF \& SVM & & & 91.6 \\
\hline & \multirow{2}{*}{ SFEW (SPI) } & Our & LBP+FAP \& SVM & $x$ & 7 & 26.1 \\
\hline & & {$[67]$} & LPQ+PHOG \& SVM & - & 4 & 19.0 \\
\hline
\end{tabular}




\begin{tabular}{|c|c|c|c|c|c|c|}
\hline & & {$[66]$} & HOG \& SVM & $x$ & 7 & 29.3 \\
\hline \multirow{4}{*}{ Lab-based } & \multirow{4}{*}{ NVIE } & Our & LBP+FAP \& SVM & $x$ & 6 & 81.8 \\
\hline & & {$[68]$} & $\begin{array}{l}\text { AAM \& KNN } \\
\text { PCA \& KNN }\end{array}$ & $\sqrt{ }$ & 3 & $\begin{array}{l}67.8 \\
65.3\end{array}$ \\
\hline & & [69] & AAM \& KNN & $\sqrt{ }$ & 3 & 61.1 \\
\hline & & {$[70]$} & Distance \& KNN & $\sqrt{ }$ & 3 & 57.4 \\
\hline
\end{tabular}

Note: 'Reg.', 'No.', and 'Acc.' indicate face registration, the number of classified expressions, and classification accuracy respectively.

Table 8

Performance comparisons to the BeFIT workshop baseline [67] on the SFEW SPI dataset. Figures in bold indicate that the proposed system has higher values than the baseline.

\begin{tabular}{ccccccccc}
\hline & & Anger & Disgust & Fear & Happiness & Sadness & Surprise & Neutral \\
\hline \multirow{2}{*}{ Precision } & BeFIT & 0.17 & 0.15 & 0.20 & 0.28 & 0.16 & 0.15 & 0.22 \\
& Our & $\mathbf{0 . 1 8 3}$ & $\mathbf{0 . 2 1 2}$ & $\mathbf{0 . 2 0 9}$ & $\mathbf{0 . 4 5}$ & 0.138 & $\mathbf{0 . 3 7}$ & 0.209 \\
\hline \multirow{2}{*}{ Recall } & BeFIT & 0.21 & 0.13 & 0.18 & 0.29 & 0.16 & 0.12 & 0.21 \\
& Our & $\mathbf{0 . 2 4 4}$ & $\mathbf{0 . 1 4 7}$ & $\mathbf{0 . 1 8 2}$ & $\mathbf{0 . 5 1 6}$ & 0.056 & $\mathbf{0 . 2 8 2}$ & $\mathbf{0 . 3 2 2}$ \\
\hline \multirow{2}{*}{ Specificity } & BeFIT & 0.48 & 0.66 & 0.64 & 0.51 & 0.60 & 0.66 & 0.61 \\
& Our & $\mathbf{0 . 6 0 1 9}$ & $\mathbf{0 . 8 1 9 3}$ & $\mathbf{0 . 7 0 7 9}$ & $\mathbf{0 . 5 5 9 5}$ & $\mathbf{0 . 8 2 1 4}$ & $\mathbf{0 . 7 6 1 9}$ & 0.5043 \\
\hline
\end{tabular}

\section{Practical Applications}

This section applies the IED system to provide insights into an image subset from the British Broadcast Corporation (BBC) regarding the presence of faces and facial expressions. It demonstrates the potential of using the human face towards affective labeling, classification, and retrieval of realistic multimedia data in practical applications, which has not been fully exploited by existing studies.

\subsection{Dataset and Problem Description}

The BBC's ELVIS image dataset ${ }^{2}$ is a large archive that covers a wide range of topics and occasions, from news photography to in-house studio shots of actors. Many pictures were taken from before the existence of the Internet, reaching back to 1900s, which are rare in other databases. To enable search and re-use, images are all labeled with a title, a short human made description, and copyright information. If relevant for a specific picture, a location or personality field is also filled. A problem with this dataset however is the absence of any ground truth data relating to the presence of faces or their emotional expression, so that the

\footnotetext{
2 The dataset is not freely available, academic institutions interested in working with it should contact jana.eggink@bbc.co.uk, license agreements might be available for collaborative work between the BBC and individual universities.
} 
proposed IED system could be of practical use here in providing insights into such ground truths.

A subset of 5,000 images is randomly selected and used for the experimental evaluation in this paper. In most cases there are one or more people in the picture, but the subset also includes other images such as those of the recently discovered skeleton of the English king Richard the III, where no actual person is visible. Images without any detected faces or with multiple faces were excluded as they make manual inspection of the results harder.

\subsection{First Impressions}

The number of images is too large to assess classification results for all images individually. For a first impression, we sorted the pictures according to their likelihood in each of the three emotion classes, positive, negative, and neutral. The most positive faces were very convincingly happily smiling; while the least positive ones were looking very surly and unhappy, see Figs. 12a and b.

The neutral class overlapped with some of the positive class, and most neutral faces were identical to the least positive ones. Most of negative faces were more problematic, containing many instances of people talking with an open mouth, such as politicians giving a speech or taking part in a debate as shown in Fig. 12c. In the authors' opinion, these faces were mostly neutral rather than negative. The negative class also contained many false positives from the face detection stage. It seems that these incorrectly identified faces often have a slightly 'unusual' arrangement of facial features, particularly the mouth region.

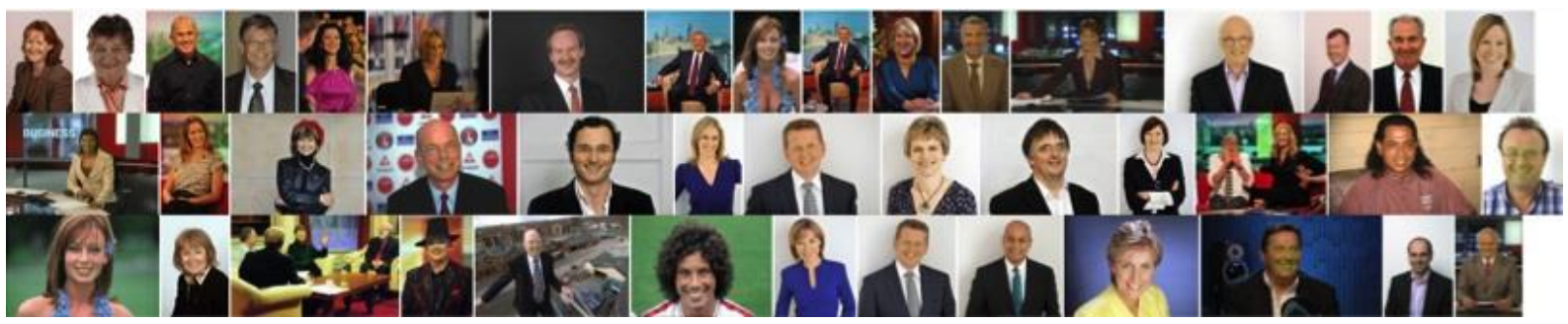

(a) Most positive images

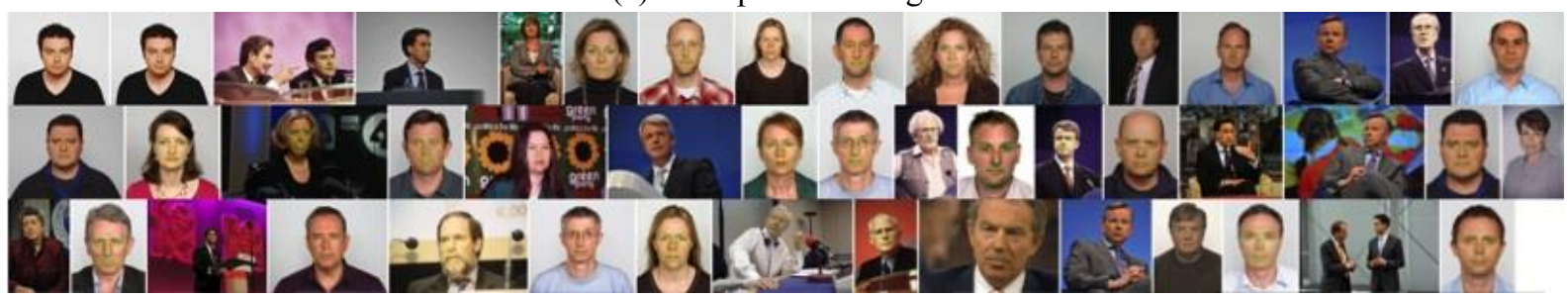

(b) Least positive images 


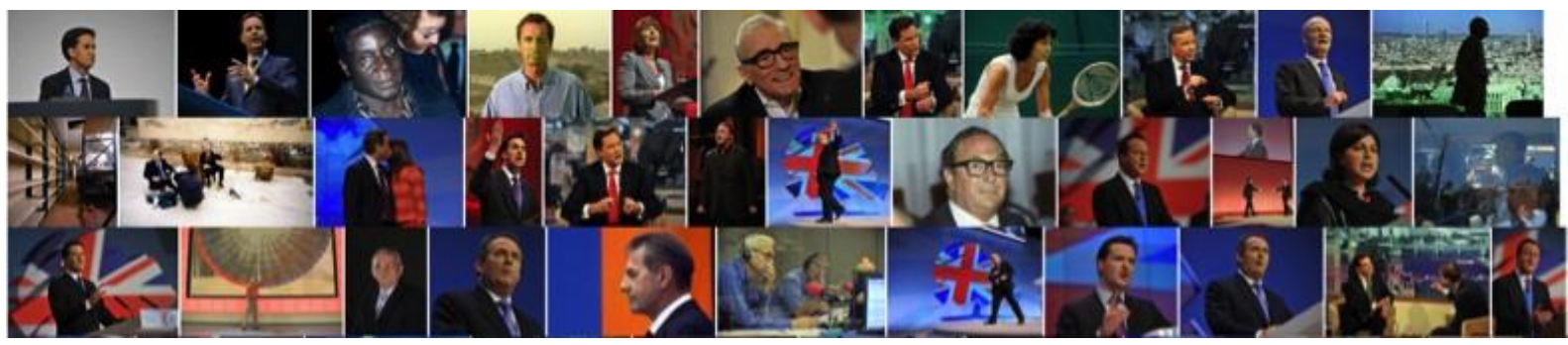

(c) Samples of incorrectly identified faces

Fig. 12. Samples of the classification results using the proposed IED system on BBC images.

To illustrate the impact of the mouth region on the performance, we compare the distributions of the height of the mouth region between images from positive, neutral and negative emotions as shown in Fig. 13. Table 9 shows the differences in the mean and standard deviation between the distributions. The height of the mouth region is calculated as the distance between top and bottom points of the lip (i.e. points indexed 52 and 58 in Fig. 2a) normalized by the distance between the two eyes. As can be seen, positive emotion tends to have the highest openness of the mouth, which is followed by negative while neutral has the smallest. This explains our result that it is more challenging to distinguish negative from positive or neutral, because a negative image with an open mouth might actually belong to positive or neutral. However, there is no significant difference in standard deviations between the distributions.

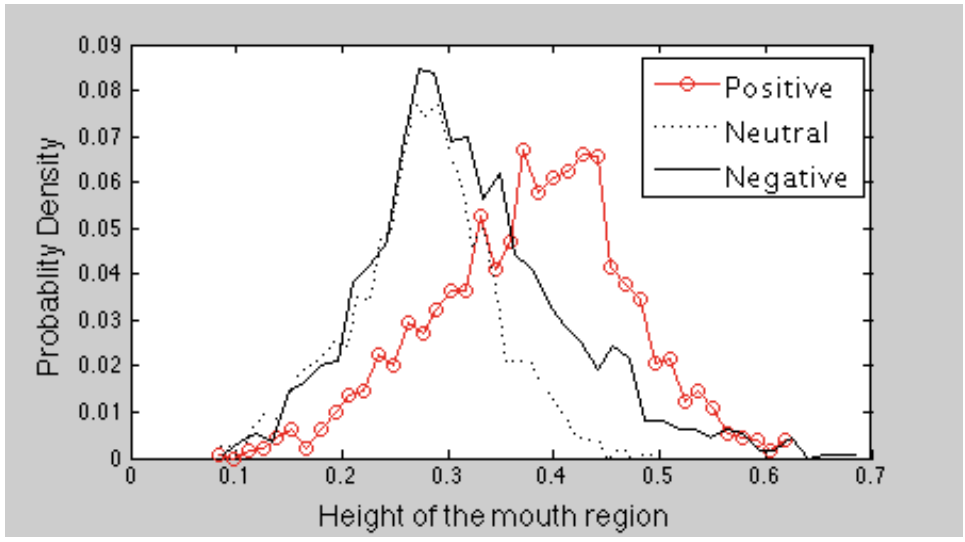

Fig. 13. Distributions of the height of the mouth region in positive, neutral and negative images.

Table 9

Mean and standard deviation of the distributions.

\begin{tabular}{cccc}
\hline & Positive & Neutral & Negative \\
\hline Mean & 0.3779 & 0.2761 & 0.3194 \\
Std. Dev. & 0.0923 & 0.0673 & 0.0952 \\
\hline
\end{tabular}




\subsection{Further Insights}

This informal analysis of the results revealed that images of a person speaking were often misclassified due to the open mouth position. In realistic applications that work with large datasets like precision is often more important than recall, as the user will immediately see misclassified images, but is less likely to notice 'missing' images in a particular emotion class. Automatically identifying faces with an open mouth for emotion classification is still a challenge, but very helpful for not losing a user's trust into the system. In addition to the emotion classification, a system to detect faces with an open mouth due to speaking was trained:

$$
\begin{gathered}
R=\text { Height }_{\text {mouth }} / \text { Widt }_{\text {mouth }} \\
\text { Face }=\left\{\begin{array}{cc}
\text { Open Mouth } & \text { if } 0.55<R<1 \\
\text { Emotional } & \text { if } R \leq 0.55 \text { or } R \geq 1
\end{array}\right.
\end{gathered}
$$

where, Height $t_{\text {mouth }}$ and Width $h_{\text {mouth }}$ represent the height and the width of the mouth region respectively. The threshold values of 0.55 and 1 are set based on preliminary experimental results.

Out of the dataset of 5,000 images, 246 were classified as having an open mouth. Manual inspection showed that precision was very good, if the face and facial feature point detection were correct, so was the classification of there being an open mouth in nearly all cases. A few images showed people laughing with an open mouth, and while in a strict sense they were correctly classified as having an open mouth, from an application point of view they would ideally be classified as having a positive expression instead - even though missing a face with a positive expression would be acceptable to most application if it noticeable improves overall precision. A relatively large number of false positives from the face detection were also classified as having an open mouth. Overall, the number of detected open mouth faces was relatively small, and recall was hard to approximate on this unlabeled dataset. The influence on the results of the negative emotion class was also relatively small, leading to an overall unconvincing impression for this class. This suggests the need for further targeted efforts to distinguish negative from other emotions.

\section{Conclusion}

This paper presents an automatic image emotion detector (IED) that is capable of classifying affective states from facial images captured from uncontrolled environments with 
variations in illumination, pose, face size and registration error etc. To achieve the best performance in both accuracy and computation, experiments are conducted to compare a set of key parameters of the system. Experiments on the GENKI-4K and QUT-FER image datasets, and performance comparisons on realistic and laboratory-based data demonstrated state-of-the-art accuracy and robustness to realistic pose variations. The proposed system has real-time processing speed on the GENKI-4K dataset and can process more than 10 images per second on the QUT-FER dataset.

The experiment with $\mathrm{BBC}$ image subset was crucial to provide insight into the practical opportunities and challenges with facial expression recognition. Preliminary results confirm the feasibility of utilizing facial expression for affective image classification. The results have indicated the need to improve the proposed FER framework to include a step to distinguish negative from other emotions and recognize the state-of-the-mouth (e.g. open mouth vs. emotional mouth), which has a big influence on the overall accuracy.

To become fully applicable for practical scenarios, the proposed system can be further improved from several aspects, by 1) implementing the system using more efficient languages (e.g. $\mathrm{C}$ and $\mathrm{C}++$ ) instead of Matlab, to achieve optimized processing speed, 2) employing more advanced algorithms (e.g. rotation invariant multiview face detection [44]) instead of the original $\mathrm{VJ}$ algorithm for more accurate detection of facial regions, 3 ) adopting recently proposed facial landmark detection models (e.g. constrained local model [48] and mixtures of parts [49]) instead of the ASM to achieve more robust facial points tracking under unconstrained environments, 4) investigating a binary classification of negative vs. the rest for more accurate recognition of negative emotion, and 5) representing appearance differences in the mouth regions with an open mouth and an emotional mouth using texture descriptors, such as LBP, for the identification of the state-of-the-mouth.

\section{Acknowledgments}

This work is funded by the British Broadcast Corporation, Australian Smart Services CRC, and the National Natural Science Foundation of China (Grant No. 61402362).

\section{References}

1. Hanjalic A (2006) Extracting moods from pictures and sounds: towards truly personalized TV. Signal Processing Magazine, IEEE 23 (2):90-100

2. Joonwhoan L, EunJong P (2011) Fuzzy Similarity-Based Emotional Classification of Color Images. Multimedia, IEEE Transactions on 13 (5):1031-1039

3. Zeng Z, Pantic M, Roisman GI, Huang TS (2009) A Survey of Affect Recognition

Methods: Audio, Visual, and Spontaneous Expressions. Pattern Analysis and Machine Intelligence, IEEE Transactions on 31 (1):39-58 
4. Zhang L, Tjondronegoro D, Chandran V (2014) Facial expression recognition experiments with data from television broadcasts and the World Wide Web. Image and Vision Computing 32 (2):107-119.

5. Jana M, Allan H Affective image classification using features inspired by psychology and art theory. In: Proceedings of the international conference on Multimedia, Firenze, Italy, 2010. ACM, pp 83-92.

6. Michela D, Pamela Z, Giulia B, Liliana A Emotion based classification of natural images. In: Proceedings of the 2011 international workshop on Detecting and Exploiting Cultural diversity on the social web, Glasgow, Scotland, UK, 2011. ACM, pp 17-22. doi: $10.1145 / 2064448.2064470$

7. Liu N, Dellandréa E, Tellez B, Chen L (2011) Associating Textual Features with Visual Ones to Improve Affective Image Classification. In: International Conference on Affective Computing and Intelligent Interaction (ACII2011), vol 6974. Lecture Notes in Computer Science. Springer Berlin / Heidelberg, pp 195-204.

8. Bianchi-Berthouze N (2003) K-DIME: an affective image filtering system. Multimedia, IEEE 10 (3):103-106

9. Hanjalic A, Li-Qun X (2005) Affective video content representation and modeling. Multimedia, IEEE Transactions on 7 (1):143-154

10. Canini L, Benini S, Leonardi R (2013) Affective Recommendation of Movies Based on Selected Connotative Features. Circuits and Systems for Video Technology, IEEE Transactions on 23 (4):636-647.

11. Acar E, Hopfgartner F, Albayrak S (2014) Understanding Affective Content of Music Videos through Learned Representations. In: Gurrin C, Hopfgartner F, Hurst W, Johansen H, Lee H, O'Connor N (eds) MultiMedia Modeling, vol 8325. Lecture Notes in Computer Science. Springer International Publishing, pp 303-314.

12. Blei DM, Ng AY, Jordan MI (2003) Latent dirichlet allocation. J Mach Learn Res 3:9931022

13. Hofmann T (2001) Unsupervised Learning by Probabilistic Latent Semantic Analysis. Machine Learning 42 (1-2):177-196.

14. Fei-Fei L, Perona P A Bayesian hierarchical model for learning natural scene categories. In: Computer Vision and Pattern Recognition, 2005. CVPR 2005. IEEE Computer Society Conference on, 20-25 June 2005 2005. pp 524-531 vol. 522.

15. Han D, Li W, Li Z (2008) Semantic image classification using statistical local spatial relations model. Multimedia Tools and Applications 39 (2):169-188.

16. Ionescu B, Schluter J, Mironica I, Schedl M A naive mid-level concept-based fusion approach to violence detection in Hollywood movies. In: Proceedings of the 3rd ACM conference on International conference on multimedia retrieval, Dallas, Texas, USA, 2013. ACM, 2461502, pp 215-222.

17. Xu M, Wang J, He X, Jin J, Luo S, Lu H (2012) A three-level framework for affective content analysis and its case studies. Multimedia Tools and Applications:1-23.

18. Mehrabian A (1968) Communication without words. Psychology Today 2 (9):52-55

19. Rudovic O, Pantic M, Patras I (2013) Coupled Gaussian processes for pose-invariant facial expression recognition. Pattern Analysis and Machine Intelligence, IEEE Transactions on 35 (6):1357-1369.

20. Ekman P (1994) Strong Evidence for Universals in Facial Expressions - A Reply to Russells Mistaken Critique. Psychol Bull 115 (2):268-287 
21. Ekman P, Friesen, W. (1978) The Facial Action Coding System: A technique for the measurement of facial movement. Consulting Psychologists Press, Palo Alto, CA, USA:274-280

22. Russell JA (1980) A circumplex model of affect. Journal of Personality and Social Psychology 39 (6):1161-1178

23. Caridakis G, Karpouzis K, Wallace M, Kessous L, Amir N (2010) Multimodal user's affective state analysis in naturalistic interaction. Journal on Multimodal User Interfaces 3 (1):49-66.

24. Anisetti M, Bellandi V (2009) Emotional State Inference Using Face Related Features. In: Damiani E, Jeong J, Howlett R, Jain L (eds) New Directions in Intelligent Interactive Multimedia Systems and Services - 2, vol 226. Studies in Computational Intelligence. Springer Berlin Heidelberg, pp 401-411.

25. Zhang L, Tjondronegoro D, Chandran V (2014) Representation of facial expression categories in continuous arousal-valence space: Feature and correlation. Image and Vision Computing 32 (12):1067-1079.

26. Maja P, Nicu S, Jeffrey FC, Thomas H (2005) Affective multimodal human-computer interaction. Paper presented at the Proceedings of the 13th annual ACM international conference on Multimedia, Hilton, Singapore,

27. Mingli S, Dacheng T, Zicheng L, Xuelong L, Mengchu Z (2010) Image Ratio Features for Facial Expression Recognition Application. Systems, Man, and Cybernetics, Part B: Cybernetics, IEEE Transactions on 40 (3):779-788

28. Kotsia I, Zafeiriou S, Pitas I (2008) Texture and shape information fusion for facial expression and facial action unit recognition. Pattern Recognition 41 (3):833-851

29. Xie X, Lam K-M (2009) Facial expression recognition based on shape and texture. Pattern Recognition 42 (5):1003-1011

30. Sung J, Kim D (2008) Pose-Robust Facial Expression Recognition Using View-Based 2D+3D AAM. Systems, Man and Cybernetics, Part A: Systems and Humans, IEEE Transactions on 38 (4):852-866

31. Ashraf AB, Lucey S, Cohn JF, Chen T, Ambadar Z, Prkachin KM, Solomon PE (2009) The painful face - Pain expression recognition using active appearance models. Image and Vision Computing 27 (12):1788-1796

32. Tsalakanidou F, Malassiotis S (2010) Real-time 2D+3D facial action and expression recognition. Pattern Recognition 43 (5):1763-1775

33. Yongmian Z, Qiang J (2005) Active and dynamic information fusion for facial expression understanding from image sequences. Pattern Analysis and Machine Intelligence, IEEE Transactions on 27 (5):699-714

34. Panning A, Al-Hamadi A, Niese R, Michaelis B (2008) Facial expression recognition based on Haar-like feature detection. Pattern Recognition and Image Analysis 18 (3):447452

35. Zisheng L, Jun-ichi I, Kaneko M Facial-component-based bag of words and PHOG descriptor for facial expression recognition. In: Systems, Man and Cybernetics, 2009. SMC 2009. IEEE International Conference on, 11-14 Oct. 2009 2009. pp 1353-1358

36. Zhengyou Z, Lyons M, Schuster M, Akamatsu S Comparison between geometry-based and Gabor-wavelets-based facial expression recognition using multi-layer perceptron. In: Automatic Face and Gesture Recognition, 1998. Proceedings. Third IEEE International Conference on, 1998. pp 454-459 
37. Peng W, Kohler C, Barrett F, Gur R, Verma R Quantifying Facial Expression Abnormality in Schizophrenia by Combining 2D and 3D Features. In: Computer Vision and Pattern Recognition, 2007. CVPR '07. IEEE Conference on, 2007. pp 1-8

38. Feng X, Lai Y, Mao X, Peng J, Jiang X, Hadid A (2013) Extracting Local Binary Patterns from Image Key Points: Application to Automatic Facial Expression Recognition. In: Kämäräinen J-K, Koskela M (eds) Image Analysis, vol 7944. Lecture Notes in Computer Science. Springer Berlin Heidelberg, pp 339-348.

39. Viola P, Jones MJ (2004) Robust Real-Time Face Detection. International Journal of Computer Vision 57 (2):137-154

40. Cootes TF, Taylor CJ, Cooper DH, Graham J (1995) Active Shape Models-Their Training and Application. Comput Vis Image Underst 61 (1):38-59

41. Mita T, Kaneko T, Hori O Joint Haar-like features for face detection. In: Computer Vision, 2005. ICCV 2005. Tenth IEEE International Conference on, 2005. pp 1619-1626 Vol. 1612

42. Anisetti M, Bellandi V, Damiani E, Arnone L, Rat B (2008) A3FD: Accurate 3D Face Detection. In: Damiani E, Yétongnon K, Schelkens P, Dipanda A, Legrand L, Chbeir R (eds) Signal Processing for Image Enhancement and Multimedia Processing, vol 31. Multimedia Systems and Applications Series. Springer US, pp 155-165.

43. Li S, Zhu L, Zhang Z, Blake A, Zhang H, Shum H (2002) Statistical Learning of Multiview Face Detection. In: Computer Vision - ECCV 2002. pp 117-121

44. Chang H, Haizhou A, Yuan L, Shihong L (2007) High-Performance Rotation Invariant Multiview Face Detection. Pattern Analysis and Machine Intelligence, IEEE Transactions on 29 (4):671-686

45. Zhang C, Zhang Z (2010) A survey of recent advances in face detection. Technical Report, Microsoft Research,

46. Milborrow S, Nicolls F (2008) Locating Facial Features with an Extended Active Shape Model. In: Forsyth D, Torr P, Zisserman A (eds) Computer Vision - ECCV 2008, vol 5305. Lecture Notes in Computer Science. Springer Berlin Heidelberg, pp 504-513.

47. Anisetti M, Bellandi V, Damiani E, Beverina F 3D Expressive Face Model-based Tracking Algorithm. In: Signal Processing, Pattern Recognition, and Applications, Innsbruck, 2006. pp 111-116

48. Chew SW, Lucey P, Lucey S, Saragih J, Cohn JF, Matthews I, Sridharan S (2012) In the Pursuit of Effective Affective Computing: The Relationship Between Features and Registration. Systems, Man, and Cybernetics, Part B: Cybernetics, IEEE Transactions on 42 (4):1006-1016.

49. Xiangxin Z, Ramanan D Face detection, pose estimation, and landmark localization in the wild. In: Computer Vision and Pattern Recognition (CVPR), 2012 IEEE Conference on, 16-21 June 2012 2012. pp 2879-2886.

50. Wu Y, Ji Q (2014) Discriminative Deep Face Shape Model for Facial Point Detection. International Journal of Computer Vision:1-17.

51. Ojala T, Pietikainen M, Maenpaa T (2002) Multiresolution gray-scale and rotation invariant texture classification with local binary patterns. Pattern Analysis and Machine Intelligence, IEEE Transactions on 24 (7):971-987

52. Hanchuan P, Fuhui L, Ding C (2005) Feature selection based on mutual information criteria of max-dependency, max-relevance, and min-redundancy. Pattern Analysis and Machine Intelligence, IEEE Transactions on 27 (8):1226-1238 
53. Tariq U, Kai-Hsiang L, Zhen L, Xi Z, Zhaowen W, Vuong L, Huang TS, Xutao L, Han TX Emotion recognition from an ensemble of features. In: Automatic Face \& Gesture Recognition and Workshops (FG 2011), 2011 IEEE International Conference on, 21-25 March 2011 2011. pp 872-877.

54. Zhang L, Tjondronegoro D, Chandran V Discovering the Best Feature Extraction and Selection Algorithms for Spontaneous Facial Expression Recognition. In: 2012 IEEE International Conference on Multimedia \& Expo (ICME 2012), 2012. pp 1027-1032

55. Shan C, Gritti T Learning discriminative lbp-histogram bins for facial expression recognition. In: Proc. British Machine Vision Conference, 2008.

56. Zhang L, Tjondronegoro D, Chandran V Evaluation of Texture and Geometry for Dimensional Facial Expression Recognition. In: Digital Image Computing Techniques and Applications (DICTA), 2011 International Conference on, 6-8 Dec. 2011 2011. pp 620-626

57. Pandzic IS, Forchheimer R (2002) MPEG-4 facial animation: the standard, implementation and applications. Wiley,

58. Hao T, Huang TS 3D facial expression recognition based on automatically selected features. In: Computer Vision and Pattern Recognition Workshops, 2008. CVPRW '08. IEEE Computer Society Conference on, 2008. pp 1-8

59. Lajevardi S, Hussain Z (2011) Automatic facial expression recognition: feature extraction and selection. Signal, Image and Video Processing:1-11.

60. Cortes C, Vapnik V (1995) Support-vector networks. Machine Learning 20 (3):273-297

61. Chang CC, Lin CJ (2001) LIBSVM: a library for support vector machines, 2001. Software available at http://wwwcsientuedutw/cjlin/libsvm

62. Whitehill J, Littlewort G, Fasel I, Bartlett M, Movellan J (2009) Toward Practical Smile Detection. Pattern Analysis and Machine Intelligence, IEEE Transactions on 31 (11):2106-2111

63. Caifeng S (2012) Smile detection by boosting pixel differences. Image Processing, IEEE Transactions on 21 (1):431-436. doi:10.1109/tip.2011.2161587

64. An L, Yang S, Bhanu B (2015) Efficient smile detection by Extreme Learning Machine. Neurocomputing 149, Part A (0):354-363.

65. Danisman T, Bilasco IM, Martinet J, Djeraba C (2013) Intelligent pixels of interest selection with application to facial expression recognition using multilayer perceptron. Signal Processing 93 (6): 1547-1556.

66. Liu M, Li S, Shan S, Chen X (2013) Enhancing Expression Recognition in the Wild with Unlabeled Reference Data. In: Lee K, Matsushita Y, Rehg J, Hu Z (eds) Computer Vision - ACCV 2012, vol 7725. Lecture Notes in Computer Science. Springer Berlin Heidelberg, pp 577-588.

67. Dhall A, Goecke R, Lucey S, Gedeon T Static facial expression analysis in tough conditions: Data, evaluation protocol and benchmark. In: Computer Vision Workshops (ICCV Workshops), 2011 IEEE International Conference on, 6-13 Nov. 2011. pp 21062112

68. Shangfei W, Zhilei L, Siliang L, Yanpeng L, Guobing W, Peng P, Fei C, Xufa W (2010) A Natural Visible and Infrared Facial Expression Database for Expression Recognition and Emotion Inference. Multimedia, IEEE Transactions on 12 (7):682-691

69. Zhaoyu W, Shangfei W Spontaneous facial expression recognition by using feature-level fusion of visible and thermal infrared images. In: Machine Learning for Signal Processing (MLSP), 2011 IEEE International Workshop on. pp 1-6 
70. Shan H, Shangfei W, Yanpeng L Spontaneous Facial Expression Recognition Based on Feature Point Tracking. In: Image and Graphics (ICIG), 2011 Sixth International Conference on, 12-15 Aug. 2011. pp 760-765 